\title{
The conserved DNMT1-dependent methylation regions in human cells are vulnerable to neurotoxicant rotenone exposure
}

\author{
Dana M. Freeman ${ }^{1}$ (D), Dan Lou' ${ }^{1}$, Yanqiang Li ${ }^{1}$, Suzanne N. Martos ${ }^{1}$ and Zhibin Wang ${ }^{1,2,3^{*}}$
}

\begin{abstract}
Background: Allele-specific DNA methylation (ASM) describes genomic loci that maintain CpG methylation at only one inherited allele rather than having coordinated methylation across both alleles. The most prominent of these regions are germline ASMs (gASMs) that control the expression of imprinted genes in a parent of origin-dependent manner and are associated with disease. However, our recent report reveals numerous ASMs at non-imprinted genes. These non-germline ASMs are dependent on DNA methyltransferase 1 (DNMT1) and strikingly show the feature of random, switchable monoallelic methylation patterns in the mouse genome. The significance of these ASMs to human health has not been explored. Due to their shared allelicity with gASMs, herein, we propose that non-traditional ASMs are sensitive to exposures in association with human disease.

Results: We first explore their conservancy in the human genome. Our data show that our putative non-germline ASMs were in conserved regions of the human genome and located adjacent to genes vital for neuronal development and maturation. We next tested the hypothesized vulnerability of these regions by exposing human embryonic kidney cell HEK293 with the neurotoxicant rotenone for $24 \mathrm{~h}$. Indeed,14 genes adjacent to our identified regions were differentially expressed from RNA-sequencing. We analyzed the base-resolution methylation patterns of the predicted non-germline ASMs at two neurological genes, HCN2 and NEFM, with potential to increase the risk of neurodegeneration. Both regions were significantly hypomethylated in response to rotenone.

Conclusions: Our data indicate that non-germline ASMs seem conserved between mouse and human genomes, overlap important regulatory factor binding motifs, and regulate the expression of genes vital to neuronal function. These results support the notion that ASMs are sensitive to environmental factors such as rotenone and may alter the risk of neurological disease later in life by disrupting neuronal development.
\end{abstract}

Keywords: DNA methylation, Allele-specific methylation, Rotenone, Parkinson's disease, Neurotoxicity

\section{Background}

DNA methylation refers to the addition of a methyl group (CH3) to the cytosine base of DNA by DNA methyltransferases. This predominately occurs at cytosine-guanine adjacent sites known as CpG sites. For most genomic

\footnotetext{
*Correspondence: zwang47@jhu.edu

${ }^{1}$ Laboratory of Environmental Epigenomes, Department

of Environmental Health \& Engineering, Bloomberg School of Public Health, Johns Hopkins University, Baltimore, MD, USA

Full list of author information is available at the end of the article
}

loci, DNA methylation is coordinated across both inherited alleles. However, some loci maintain CpG methylation at only one allele and these regions are described to have allele-specific methylation (ASM; previously known as differentially methylated region DMR) [6]. The most well-known of these regions are germline ASMs which control the expression of imprinted genes in a parent of origin-dependent manner. Imprinted genes are crucial in development and are commonly associated with genetic disorders such as Beckwith-Wiedemann, Angelman, and 
Prader-Willi syndromes [10]. In addition to the control of imprinted gene expression, DNA methylation is a key to maintain genome stability via silencing retrotransposons $[11,74]$.

Investigations demonstrate that two types of genomic regions, imprinted germline ASMs and intracisternal A-particle (IAP)-like retrotransposons, seem vulnerable to environmental factors. Therefore, these two regions are proposed to be pivotal for understanding human disease in response to exposure and popularly pursued in animal and epidemiological studies [32, 51]. The former is attractive because exposure altered ASMs are anticipated to be faithfully transmitted to somatic cells during rounds of global demethylation and remethylation in early embryos $[5,33]$. As a result, parental exposure can be epigenetically inherited to modify offspring phenotype [22]. The latter is exemplified in mice by the bisphenol A-hypomethylated IAP at the agouti gene for variations of coat color and obesity, as well as by altered methylation of IAPs at $A x i n^{F u}$ for tail kinkiness [17, 61, 83].

In our recent work, we developed two approaches, no-rescued DMRs (NORED) and methylation mosaicity analyses (MethylMosaic), to identify numerous genomic loci bearing potential ASMs [48]. Both the known imprinted germline ASMs and newly identified ASMs are dependent on DNA methyltransferase 1 (DNMT1) [44]. Many of these novel ASMs are presumably sequence (single nucleotide polymorphism; SNP)-influenced ASMs [35]. For example, in a reciprocal cross between 129S1/SvlmJ and Cast/EiJ or between C57BL/6NJ and Cast/EiJ, the Cast allele with SNP C of Hcn2 ASM is always hypomethylated (i.e., independent of parental origin), whereas the 129 allele or the C57 allele with SNP A is always hypermethylated. Standing out of the previously appreciated sequence-dependent ASMs, a new paradigm of switchable ASMs that shows equal chances of either paternal or maternal allele to be methylated was revealed by our report [48]. Importantly, the switchable feature seems also conserved in the human genome. At the DLGAP2 locus, independent evidence confirms a maternally imprinted ASM during pre-implantation switched to a random ASM in somatic tissues during gestation [50]. Collectively, the mouse genome or human genome contains more ASMs (including both sequence-dependent and switchable ASMs) than previously appreciated $[15,48,50,55]$. The newly revealed random, switchable ASMs remind us of features in X chromosome inactivation, leading to a proposed hypothesis of regional autosomal chromosome inactivation [76].

Currently, germline ASMs are being increasingly considered in human disease; however, less studied are nongermline ASMs, which maintain CpG methylation at one allele independent of the parent of origin [15, 48, 82].
These regions regulate the expression of non-imprinted genes and these genes are hypothesized to have random monoallelic expression. Due to their predicted monoallelicity (DNA methylation and transcripts), we hypothesize that these regions are also targets for environmental factors and associated with disease like germline ASMs $[34,70]$.

The goal of this study was to determine whether our identified candidate ASMs in the mouse genome were in conserved regions of the human genome and to explore the possible adverse effects of differential methylation in these regions by examining their tissue expressivity and functional enrichment. Last, we tested our hypothesis that genes adjacent to non-germline ASMs would be vulnerable to environmental factors by exposing human embryonic kidney HEK293 cells to the pesticide rotenone for $24 \mathrm{~h}$. We used whole transcriptome RNA-sequencing and targeted bisulfite-amplicon sequencing to evaluate changes in expression of adjacent genes and DNA methylation at candidate ASMs in response to rotenone. Indeed, our data demonstrate the vulnerability of these new non-traditional ASMs to environmental exposure [23].

\section{Results \\ DNMT1-dependent regions in the mouse and human genome}

Two approaches, NORED and MethylMosaic, independently identified over 2000 regions with DNMT1 dependency and allele-specific methylation. To simplify future interpretation, we focused on 207 overlapped regions (i.e., 'NORED + MethylMosaic' regions) to initiate our investigation. We compared these 207 regions from mouse and observed 145 of these regions were conserved in the human genome. Most regions identified in the human genome were highly conserved with $>70 \%$ matched bases for more than $90 \%$ of the entire span of the region (Fig. 1a, b). Analyzing these regions in the genome browser, we noted that $70 \%$ of the conserved regions in the human genome were located in the gene body and approximately 50\% of them had transcription factor binding sites in human embryonic stem cells (Fig. 1c, d). The two transcription factors that were the most significantly enriched were POL2RA and TAF1 with binding sites at $19 \%$ of conserved DNMT1 regions. Both were concentrated around transcription start sites and regulate RNA polymerase II binding and processivity in gene transcription. The third most enriched transcription factor was CTCF with binding motifs in $17 \%$ of conserved regions and most often found within intragenic regions. The top transcription factors with binding motifs found in intergenic sites were CTCF, SIN3A, and RAD21. All three transcription factors are crucial in 
a

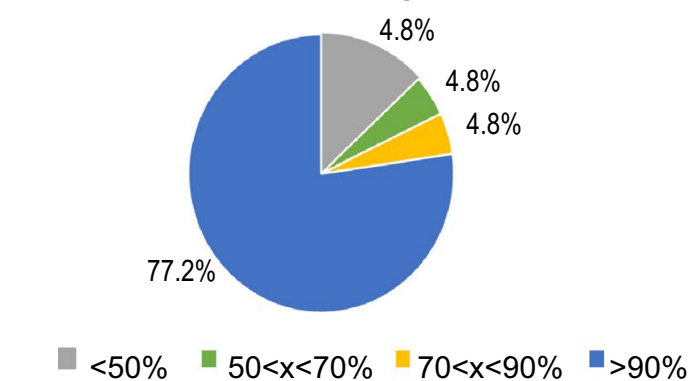

b

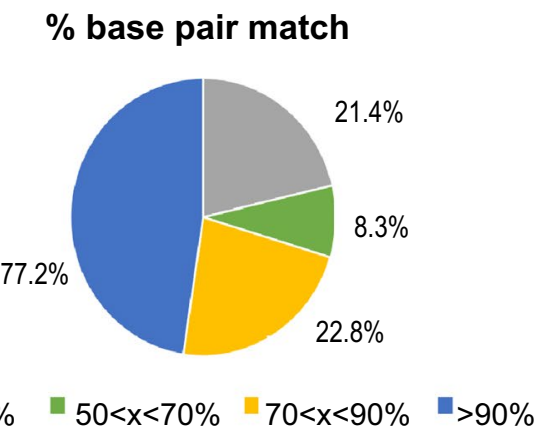

C

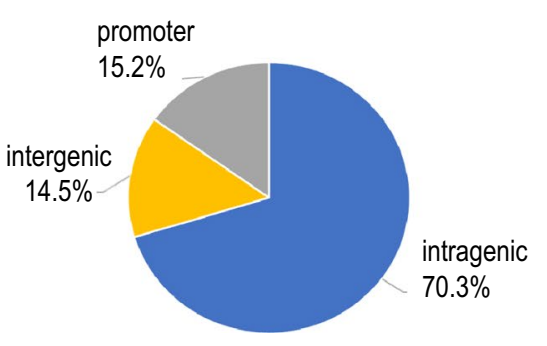

d

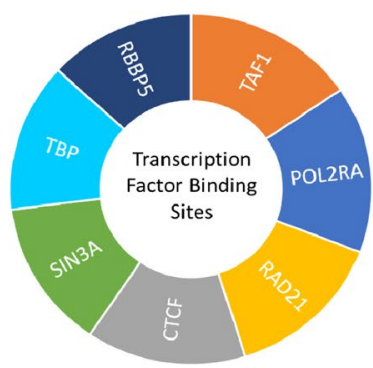

e

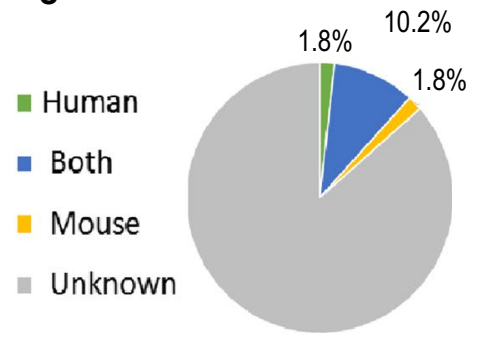

$88.0 \%$

Fig. 1 Characterization of conserved DNMT1-dependent regions in the Human genome. a The percent span of the DNMT1-dependent regions in mouse covered by the identified human conserved regions. Pie chart represents the percentage of all identified conserved regions in the human genome that fall into each category. b The percent base-pair match of the DNMT1-dependent regions in mouse with the identified human conserved regions. Pie chart represents the percentage of all conserved regions in the human genome that fall into each category. $\mathbf{c}$ The percentage of all conserved regions in the human genome that are located within the promoter, the gene body, or in non-coding intergenic regions. $\mathbf{d}$ The top transcription factor binding sites found within all human conserved regions. $\mathbf{e}$ The percentage of known germline ASMs in our conserved DNMT1-dependent regions separated by genome

regulating chromatin structure to repress gene transcription and inhibition of these factors is closely associated with human disease $[14,78,85]$. We searched conserved human genes for known imprinted genes at germline ASMs using the GeneImprint database and found 20 known imprinted genes (Fig. 1e). Most of the human genes found at conserved DNMT1-dependent regions had an unknown imprinted status and thus were considered candidate non-germline ASMs. Prior examination of DNA methylation in four independent mouse embryonic stem cell lines validated our hypothesis at one conserved gene (HCN2) that non-germline ASMs can exhibit a random, switchable pattern [48].

\section{Human DNMT1-dependent genes are enriched in cellular processes associated with cell-cell signaling}

Out of the 145 regions identified in the human genome, we selected 97 of the most highly conserved regions compared to the mouse genome. The genes nearest to these regions on the same allele (112 genes) were used for functional enrichment analyses (Table 1). We used Gene Ontology functional annotations to gain insights into the cellular processes associated with these genes and significance was determined from the Fisher's exact test with $p$ value adjustment using false-discovery rate method (FDR $<0.05)$. We observed adjacent genes were highly associated with cell to cell interactions and signaling. The number of genes involved in this biological process as well as the significance of its enrichment (expressed as log base 2 false-discovery rate) are shown in Fig. 2a. Genes regulating cell-cell adhesion belonged primarily to the cadherin protein family. This agrees with previous reports showing monoallelic expression of protocadherins in Purkinje neurons [19]. Gene Ontology of cellular components describes the subcellular compartments where enriched cellular processes and molecular functions occur. The plasma membrane and the presynapse were significantly enriched in our dataset in accordance with the enrichment of cell-cell interactions and calcium ion binding (FDR $<0.05$, Additional file 1 : Table S3).

The interaction of the proteins encoded by DNMT1dependent genes was analyzed using the STRING database (Fig. 2b). The STRING database is a commonly used platform that summarizes the functional associations of a group of proteins. Out of the 112 selected 


Table 1 Predicted human DNMT1-dependent loci
and genes

chr1:203012544-203013111

chr9:130633077-130634324

chr20:2736325-2736555

chr20:30133018-30134961

chr20:30134986-30135324

chr20:36149063-36149960

chr20:36150645-36151532

chr20:37356947-37357083

chr20:57424303-57428479

chr20:57429454-57430628

chr20:57428494-57429364

chr20:57430647-57430802

chr1:151762641-151762866

chr1:40769328-40769695

chr1:23789986-23792384

chr1:20569708-20569903

chr1:17570695-17571208

chr4:3809685-3810214

chr4:6575638-6576768

chr4:24982328-24982857

chr1:90308879-90309031

chr1:90309136-90309398

chr12:120031502-120031941

chr12:117146822-117147291

chr12:113400413-113400788

chr7:94284623-94285630

chr7:94285746-94285993

chr7:94286061-94286219

chr7:94286263-94287973

chr7:130126204-130127042

chr7:130129122-130129865

chr7:130130320-130132940

chr7:130133202-130135402

chr7:134955283-134955477

chr7:139942121-139942907

chr4:89618037-89618988

chr3:3842722-3843091

chr12:6451268-6451802

chr12:14518500-14518687

chr19:55677470-55677864

chr19:57349655-57353647

chr19:47138150-47139513

chr19:46148498-46149609

chr19:45260696-45260939

chr19:44008046-44008221

chr19:42810915-42811210

chr11:2018645-2019501

chr11:2718814-2720223

chr11:2720461-2722038

chr4:175134957-175135614

\begin{tabular}{|c|}
\hline PPFIA4 \\
\hline$A K 1$ \\
\hline EBF4 \\
\hline MCTS2P/HM13 \\
\hline MCTS2P/HM13 \\
\hline NNAT/BLCAP \\
\hline NNAT/BLCAP \\
\hline$S L C 32 A 1$ \\
\hline GNAS \\
\hline GNAS \\
\hline GNAS \\
\hline GNAS \\
\hline TDRKH \\
\hline COL9A2 \\
\hline ASAP3 \\
\hline UBXN10NWA5B1/LINC0175 \\
\hline PAD/1 \\
\hline ADRA2C \\
\hline MAN2B2 \\
\hline CCDC149/LG/2 \\
\hline$\angle R R C 8 D$ \\
\hline$\angle R R C 8 D$ \\
\hline TMEM233 \\
\hline C12orf49 \\
\hline OAS3 \\
\hline SGCE \\
\hline PEG10 \\
\hline PEG10 \\
\hline PEG10 \\
\hline MEST \\
\hline MEST \\
\hline MEST \\
\hline MEST \\
\hline STRA8 \\
\hline KDM7A/SLC37A3 \\
\hline NAP1L5/HERC3 \\
\hline LRRN1/SUMF1 \\
\hline TNFRSFIASSCNN1A \\
\hline ATFTIP \\
\hline DNAAF3 \\
\hline PEG3/ZIM2/MIMT1 \\
\hline GNG8 \\
\hline$E M L 2 / G I P R$ \\
\hline$B C L 3$ \\
\hline PHLDB3 \\
\hline PRR19 \\
\hline H19 \\
\hline KCNQ1OT1/KCNQ1 \\
\hline KCNQ1OT1/KCNQ1 \\
\hline AK125257/FBXO8 \\
\hline
\end{tabular}

PPFIA4

EBF4

MCTS2P/HM13

NNAT/BLCAP

NNAT/BLCAP

SLC32A 1

GNAS

GNAS

GNAS

GNAS

TDRKH

COL9A2

ASAP3

UBXN10NWA5B1/LINC01757

PADI1

ADRA2C

MAN2B2

CCDC149/LG/2

$\angle R R C 8 D$

$\angle R R C 8 D$

TMEM233

C120rf49

SGCE

PEG10

MEST

MEST

MEST

MEST

STRAB

KDM7A/SLC37A

NAP1L5/HERC3

LRRN1/SUMF1

TNFRSFIAVSCNN1A

ATFTIP

DNAAF3

PEG3/ZIM2/MIMTI

GNG8

EML2/GIPR

$B C L 3$

PHLDB3

PRR19

KCNQ1OT1/KCNQ1

AK125257/FBXO8
Table 1 (continued)

chr16:56624355-56625029

chr16:67313281-67313411

chr16:72698159-72698950

chr16:81297176-81297683

chr16:89258400-89259894

chr12:1100277-1100455

chr19:10250933-10251636

chr11:118050777-118051248

chr15:79574796-79575831

chr3:147111981-147112692

chr6:144328582-144329817

chr19:616132-616452

chr7:50850069-50850216

chr2:63271636-63272171

chr1:228612850-228612928

chr17:10551758-10552335

chr17:75723668-75724123

chr17:76133291-76133628

chr17:80187539-80188383

chr17:80797978-80799678

chr14:74814669-74815870

chr14:101275797-101278087

chr14:101290612-101291411

chr14:101292483-101292929

chr14:101292955-101294422

chr6:656305-657021

chr6:18121790-18122423

chr9:94123015-94123883

chr13:22246327-22246913

chr8:28196773-28197118

chr8:24771898-24773551

chr8:24771655-24771790

chr13:53423771-53424148

chr3:185795915-185797093

chr6:158422346-158423188

chr6:159084156-159084536

chr5:172306505-172306903

chr6:36237727-36237965

chr6:38997730-38998017

chr2:43058554-43059906

chr4:11280093-11280328

chr18:34823790-34823894

chr5:140175896-140176159

chr5:140228130-140228418

chr5:140250568-140251167

chr5:140554155-140554621

chr18:56664106-56665166
MT3

PLEKHG4

AK201563/LINC01572

BCMO1

$\mathrm{CDH} 15$

ERC1

DNMT1

SNC2B/AMICA1

ANKRD34C

ZIC4

PLAGL1

HCN2

GRB10

EHBP1

HIST3H3/TRIM17

MYH3

LINC01987

TMC8

SLC16A3

TBCD

VRTN

MEG3/DLK1/MIR2392

MEG3

MEG3

MEG3

HUSTB/EXOC2

NHLRC1

AUH

FGF9

PNOC

NEFM

NEFM

PCDH8

ETV5

SYNJ2

SYTL3

ERG/C1

PNPLA1

DNAH8

HAAO

MIR572/HSTS31

CELF4

PCDHA2/PCDHA1

PCDHA1-9

PDDHAT-11

PCDHB7

ZNF532/OACYLP 


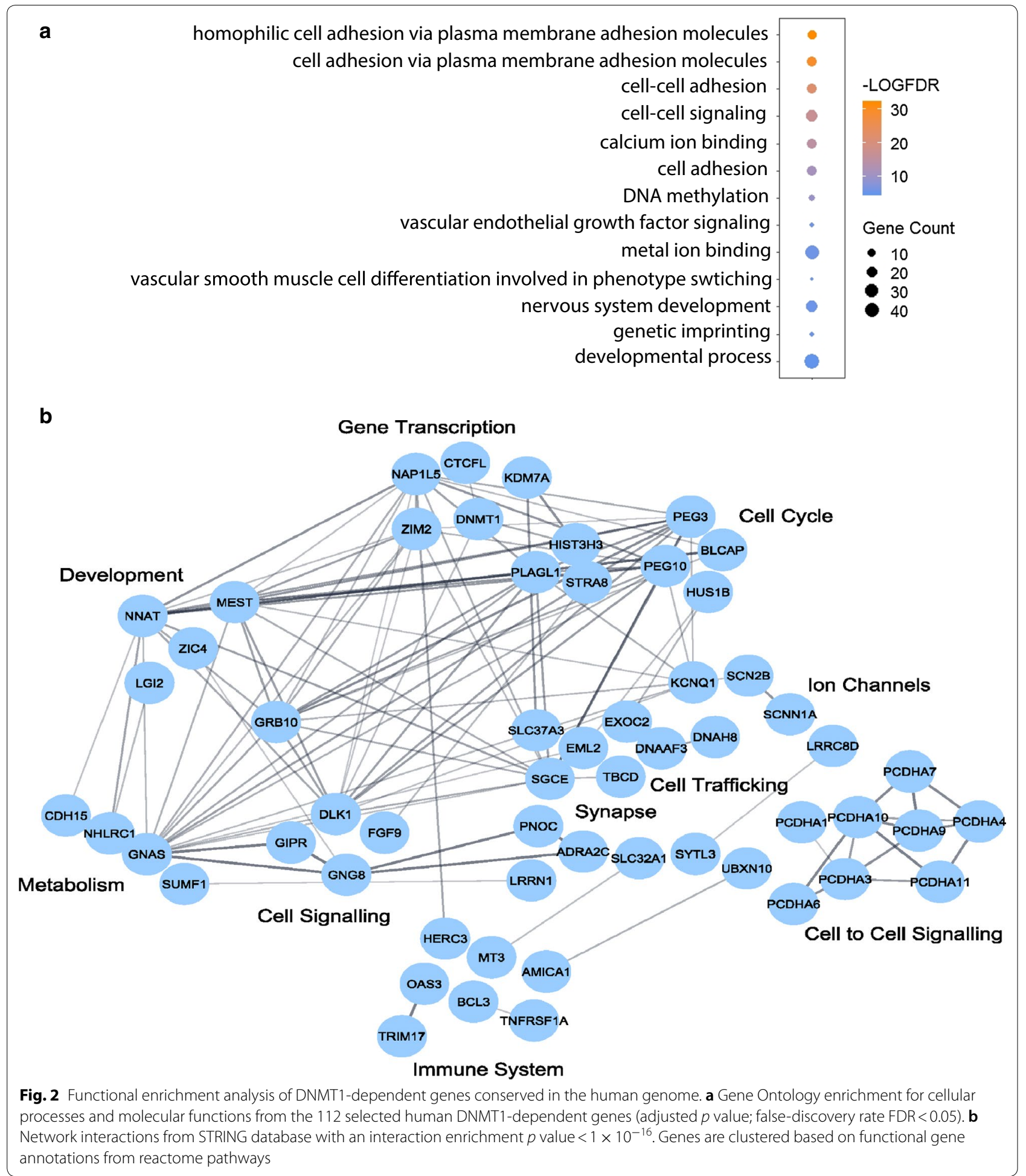

protein-encoding genes in humans, 98 nodes with 109 edges were detected with a medium confidence interaction score $(>0.4)$. The interaction $p$ value (PPI) was less than $1 \times 10^{-16}$, indicating that the number of associations was significant. We manually clustered genes with interactions using functional gene annotations. The largest cluster consisted of genes involved in cell-cell signaling including cadherins and cell surface adhesion molecules, 
cell trafficking chaperones, cytoskeleton proteins, and voltage-gated ion channels. Other significant pathways included developmental pathways of the nervous system and the vascular system.

\section{Human DNMT1-dependent genes are enriched in tissues of the brain}

Given the evidence that DNMT1-dependent genes may play an important role in cell-cell adherence and communication as well as in nervous system development, we hypothesized that DNMT1-dependent genes may be highly expressed in the brain. We analyzed the enrichment of tissue expression using the ARCHS4 human tissue database in EnrichR. The ARCHS4 database reports publicly available RNA-sequencing across all tissues and cell types in approximately 85,000 human samples [41]. Significant expression of the DNMT1-dependent genes in the adult and developing brain was observed $(p<0.01$, Fig. 3a). We also observed enrichment in the regions of the brain associated with motor function control. These structures include the cerebellum, spinal cord, and the striatum which are directly involved with motor coordination as well as the superior frontal gyrus which contains the supplementary motor area activated in complex movements [43, 67].

To further determine if these genes were specific to neuronal tissues or if they have functionality across several tissues, we analyzed DNMT1-dependent genes for tissue-specific enrichment using the TissueEnrich $\mathrm{R}$ package [31]. Genes with increased expression in one tissue compared to the expression in any other tissue were defined as tissue enriched while genes with increased expression in one tissue compared to the average of all tissues were defined as tissue enhanced. Group-enriched genes were defined as genes that have increased expression in a group of tissues compared to all other tissues. Our analysis demonstrates that DNMT1-dependent genes conserved in the human genome have a significant abundance of tissue enhanced and group-enriched genes within the brain, but not tissue-enriched genes (Fig. 3b). From these data, we conclude that DNMT1-dependent genes are likely important in cellular processes in the fetal and adult brain.

\section{Five DNMT1-dependent genes are represented in genes for potential PD blood biomarkers in patients}

To further explore the significance of identified human DNMT1-dependent genes, we evaluated the recent literature on potential blood biomarkers in Parkinson's disease (PD) patients. Encouragingly, we observed five differentially methylated genes in these studies within our conserved human regions $[29,75]$. They are COL9A2, SCNN1A, AMICA1, SLC16A3, and DLK1. One of these genes, COL9A2, was also found to be differentially expressed in our rotenone treated cells [29] (described below). Given that candidate PD biomarkers and our DNMT1-dependent genes were selected by differing criteria, we consider five overlapping genomic regions to be promising toward our hypothesis.

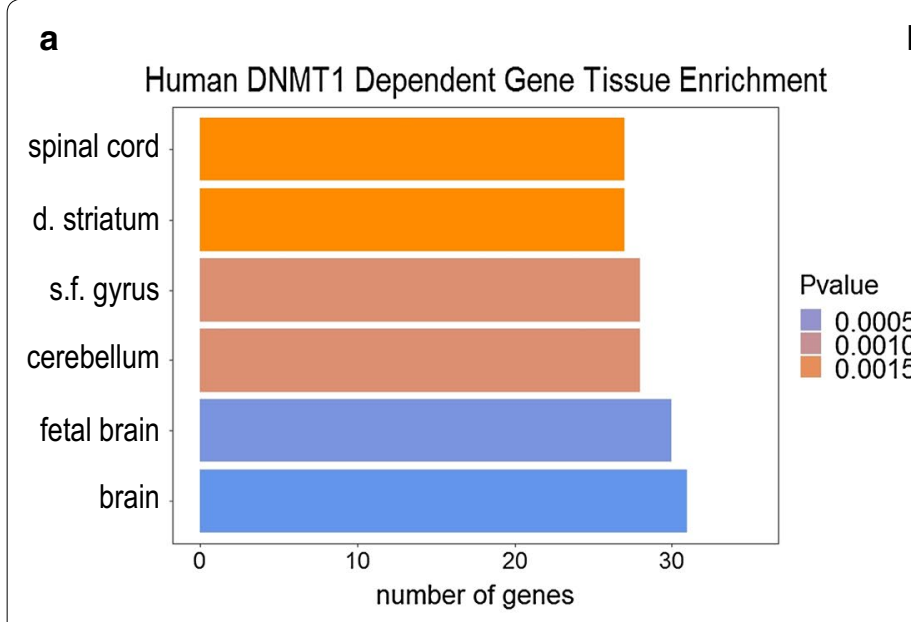

b

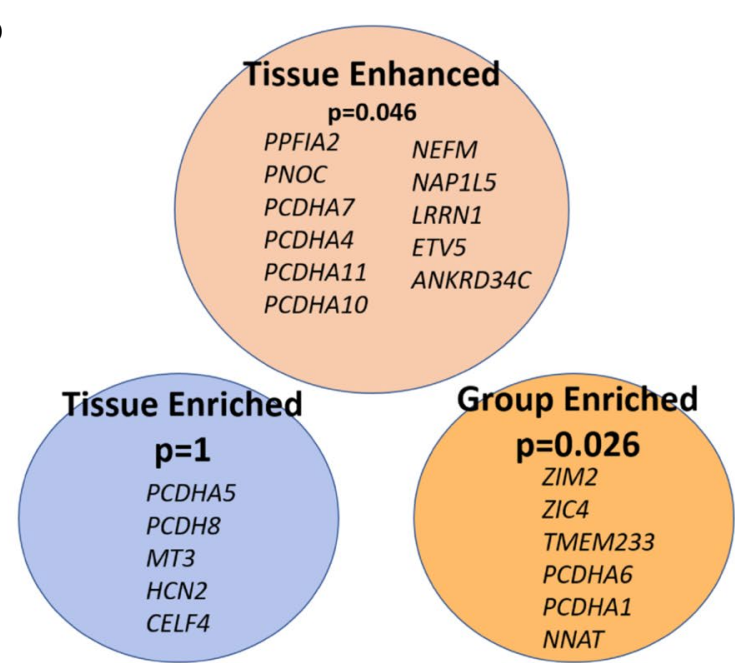

Fig. 3 Tissue enrichment analysis of DNMT1-dependent genes conserved in the human genome. a The top 6 tissues represented from the 112 selected human DNMT1-dependent genes (adjusted $p$ value < 0.01 ) scored from Enrich R using the ArchS4 human tissue database (s.f. gyrus superior frontal gyrus, $d$. striatum dorsal striatum). b DNMT1 human genes that are tissue-enriched, tissue-enhanced, or group-enriched genes within the cerebral cortex from Tissue Enrich (adjusted $p$ value shown). TissueEnrich definitions in "Methods" section 
Human DNMT1-dependent genes are differentially expressed in response to rotenone in human cells

We have shown that DNMT1-dependent regions have conserved sequences in the human genome and are enriched at genes involved in cell to cell interactions (Fig. 2). These genes have enhanced expression in the brain and may contribute to neurological dysfunction and disease in response to environmental stress (Fig. 3). To test the hypothesized contribution, we focus on rotenone exposure. Rotenone is a mitochondrial complex I inhibitor that is known to disrupt neuronal cell function in Parkinson's disease-associated brain regions [72]. These brain regions include the cerebellum, spinal cord, striatum, and basal ganglia. We treated human cell line HEK293 with rotenone $(200 \mathrm{~nm})$ for $24 \mathrm{~h}$. This dose was chosen based on previous reports in HEK293 and other neuronal cell models [28, 56, 73]. Rotenone treatment had a substantial effect on the expression levels of over 2000 genes $(\geq 1.5$-fold, FDR $\leq 0.05)$. We examined these differentially expressed genes with our identified human DNMT1-dependent genes and discovered 14 of them had been changed upon rotenone treatment (Table 2). We validated the expression of 7 of these genes with qRT$\operatorname{PCR}\left(R^{2}=0.69\right.$, Additional file 1: Figure S2).

We investigated whether these genes may contribute to rotenone-induced Parkinson's disease by observing their expression in Parkinson's disease tissues (Fig. 4). All 14 genes were expressed in Parkinson's disease regions (>1 TPM) and 8 of the genes (PPFIA4, NEFM, HCN2, ADRA2C, COL9A2, LRRC8D, EML2, and $K D M 7 A / J H D M 1 D)$ had pronounced expression in Parkinson's disease regions (>35 TPM). Two genes, NEFM and HCN2, had significant expression (>100 TPM) in all selected regions and were identified by our tissuespecific enrichment analysis as tissue enhanced and tissue enriched, respectively (Fig. 3b). Notably, Hcn2 was investigated favorably for having a switchable allele-specific methylated phenotype in our mouse model [48]. We therefore selected NEFM and HCN2 for targeted methylation analysis based on their regional expression and significant up-regulation from both RNAseq and qRT-PCR analyses. The relevance and significance of HCN2 and NEFM in human development and diseases are detailed later in "Discussion" section.

\section{DNMT1-dependent regions at HCN2 and NEFM are differentially methylated in response to rotenone in human cells}

Previously, germline ASMs are especially vulnerable to environmental exposure, thereby altering imprinted gene expression [70]. Herein, we determined the potential methylation changes of the defined DNMT1-dependent region at these two genes, NEFM and HCN2, with significant up-regulation in response to rotenone. We completed base-resolution bisulfite sequencing of these regions amplified with bisulfite PCR. After filtering of low-quality reads, approximately $42 \%$ of reads were mapped uniquely to the amplified regions. We observed high correlation between biological replicates and similar average coverage between control and treated samples (Additional file 1: Figures S2-S3). The average CpG coverage for both genes in all samples was $>15,000 \times$. Of the 23 predicted CpG sites within the amplified DNMT1dependent region on exon 8 of HCN2, 21 CpGs had

Table 2 Human DNMT1-dependent genes altered by rotenone

\begin{tabular}{|c|c|c|c|c|}
\hline Gene & $\log 2 \mathrm{FC}$ & FDR & Region type & Function/process \\
\hline MYH3 & -1.62 & $3.31 \mathrm{E}-09$ & Intragenic & Myosin protein; cell movement and transport \\
\hline PPFIA4 & -1.37 & $2.59 E-13$ & Intragenic & Neurotransmitter release synaptic function \\
\hline COL9A2 & -0.79 & $1.53 \mathrm{E}-04$ & Intragenic & Collagen; extracellular matrix organization \\
\hline DNAAF3 & -0.78 & $3.59 \mathrm{E}-02$ & Intragenic & Dynein protein assembly; cell movement \\
\hline$L R R C 8 D$ & -0.78 & $8.42 \mathrm{E}-05$ & Intragenic & Ion channel protein; neurotransmission \\
\hline PLEKHG4 & -0.65 & $2.74 \mathrm{E}-03$ & Intragenic & Guanine exchange factor; cell signaling \\
\hline$A D R A 2 C$ & 0.59 & $2.93 \mathrm{E}-02$ & Intergenic & Neurotransmitter release synaptic function \\
\hline EML2 & 0.62 & $5.99 \mathrm{E}-05$ & Promoter & Microtubule protein; synaptic function \\
\hline HCN2 & 0.66 & $1.10 \mathrm{E}-03$ & Intragenic & Voltage gated ion channel; action potential \\
\hline$K D M 7 A$ & 0.67 & $1.16 \mathrm{E}-04$ & Intergenic & Histone demethylase; neurodevelopment \\
\hline PHLDB3 & 0.76 & $3.75 \mathrm{E}-04$ & Intragenic & Enzyme binding; cell growth and proliferation \\
\hline GIPR & 0.76 & $2.37 \mathrm{E}-02$ & Promoter & Gastric inhibitory peptide; insulin release \\
\hline$B C L 3$ & 0.78 & $2.00 \mathrm{E}-02$ & Intragenic & Proto-oncogene; cell growth and proliferation \\
\hline NEFM & 0.80 & 4.33E-07 & Intragenic & Neurofilament; synaptic function \\
\hline
\end{tabular}

Log2FC is the log base twofold change of the fragments per kilobase of exon per million fragments mapped (FPKM) or "read counts" of the rotenone-treated HEK293 relative to the vehicle control. FDR is the adjusted $p$ value using the false-discovery rate correction for multiple hypotheses 


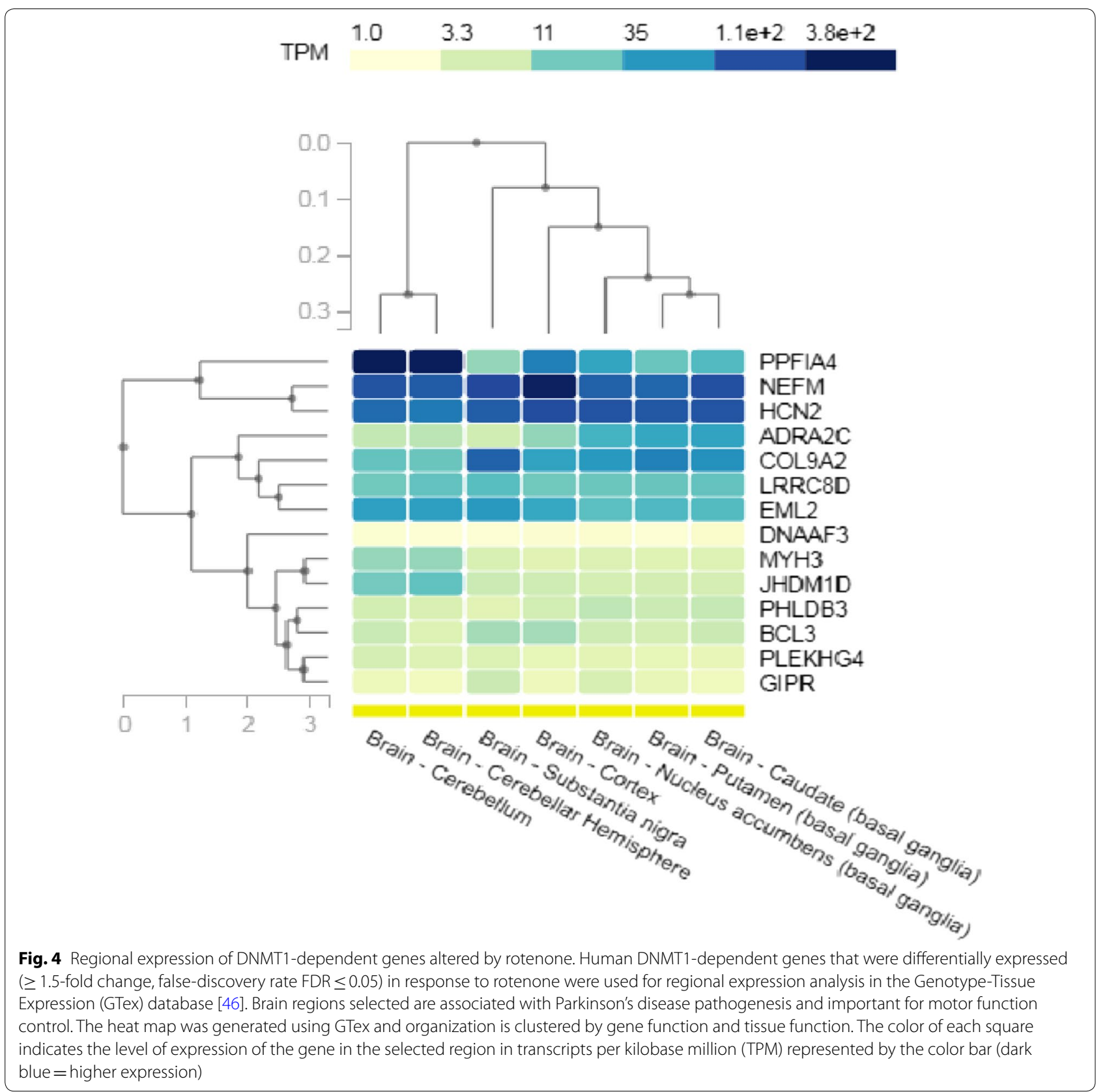

adequate coverage $(>1000 \times)$ in all samples and 14 CpGs were differentially methylated (Fig. 5; Additional file 1: Table S4). These differentially methylated cytosines were largely hypomethylated (Additional file 1: Figure S4). The mean percent methylation of all CpGs within the amplified DNMT1-dependent region was also significantly hypomethylated ( $\Delta$ me of $-1.84 \%$, FDR $<0.05)$.

We saw a similar trend in the DNMT1-dependent region at exon 1 of $N E F M$. Of the 39 predicted CpG sites within the amplified DNMT1-dependent region, 35 CpGs had adequate coverage in all samples and
13 of these CpGs were differentially methylated. A slight majority (54\%) of these differentially methylated cytosines were hypomethylated (Additional file 1: Figure S4). The overall change in methylation ratio for the entire region was significant but very low $(<0.1 \%$ absolute difference, FDR $<0.01$ ). As a result, we decided to focus on the first $200 \mathrm{bp}$ of the 500-bp amplified region, which overlap both the CpG island at exon 1 as well as a CTCF transcription factor binding site reported by ENCODE [18]. In this region, there was a slightly higher change in methylation $(\Delta \mathrm{me}$ of $-0.12 \%$, FDR $<0.05)$. In addition, 

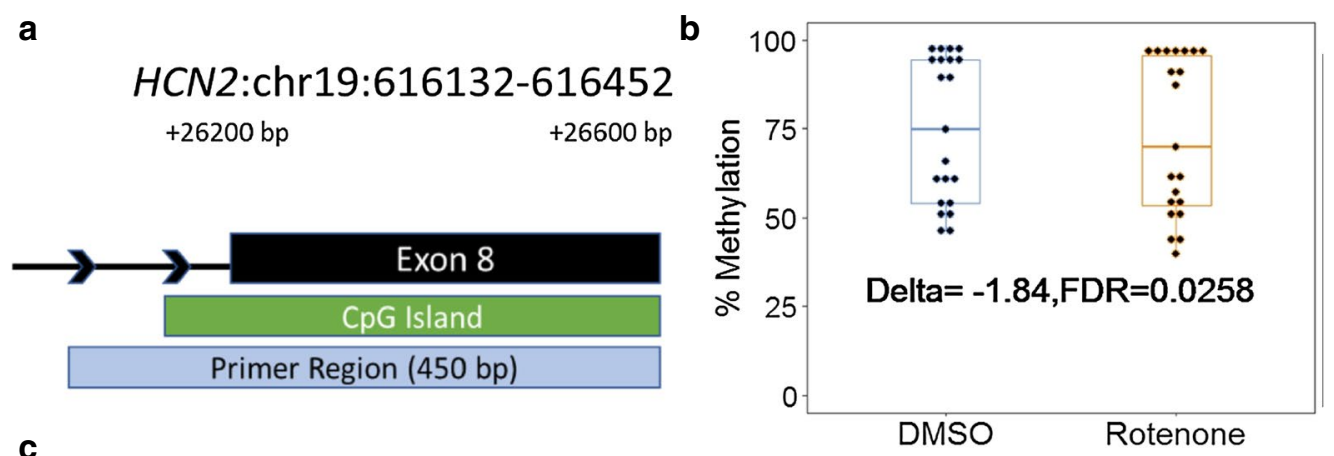

C

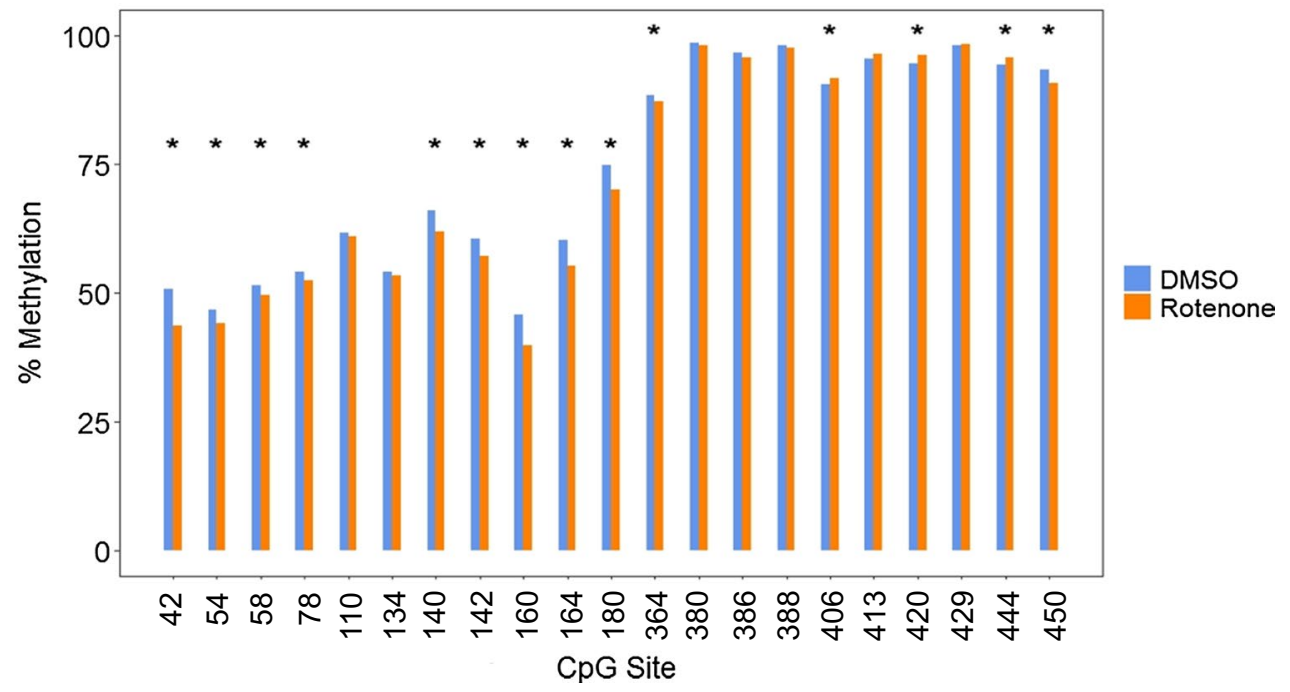

Fig. 5 Altered $\mathrm{CpG}$ methylation at HCN2 human DNMT1-dependent locus. a Genomic location of identified HCN2 DNMT1-dependent region. The DNA element and distance from the transcription start site are annotated in black. The primer region box indicates the amplified region for Bisulfite-sequencing. $\mathbf{b}$ The percent methylation of all $\mathrm{CpG}$ sites within the amplified region. Delta indicates the change in the mean CpG methylation percentage and the associated false-discovery rate. $\mathbf{c}$ The percent methylation of individual $\mathrm{CpG}$ sites within the amplified region. Significant differentially methylated cytosines are indicated by * $(\Delta>1 \%$; q value $<0.01)$

we used the CTCF binding site prediction tool to determine the exact CPG sites within the CTCF binding motif [84]. One of the top hits predicted CpG binding on the negative strand at CpG sites $89-96$ within the defined NEFM region (Fig. 6; Additional file 1: Table S5). Three of these four CpG sites were differentially methylated with half of them having $>2 \%$ reduced methylation.

These data enable us to conclude that the methylation status of DNMT1-dependent regions in the human genome is vulnerable to the neurotoxicant rotenone. We found that the coding regions and transcription factor binding motifs may be among the DNA elements that are particularly susceptible to exposure. The changes in methylation we observed were similar in scale to observed differential methylation at gene-encoding regions in the blood and brain of Parkinson's disease patients [29, 49, 52, 75]. Both HCN2 and NEFM are regionally expressed in Parkinson's disease tissues and their function has a critical role in neuronal plasticity and survival detailed in "Discussion" section.

\section{Discussion}

Our previous work identified DNMT1-dependent putative non-germline ASMs in the mouse genome. In this study, we analyzed these regions and found that $70 \%$ were in highly conserved regions with the human genome. In the human genome, our candidate loci were often located at gene-coding regions and half of them overlapped transcription factor binding sites (Fig. 1). Our observations agreed with another recent study which identified genome-wide ASMs in human samples from a Norfolk Island genetic isolate [8]. Methylated cytosines alter gene expression by influencing the binding of transcription factors to DNA. We listed the top transcription factor binding sites within our identified candidate regions in human embryonic stem cells using ENCODE experimental data (Fig. 1). Unsurprisingly, 


\section{a}

NEFM: chr8:24771898-24773551

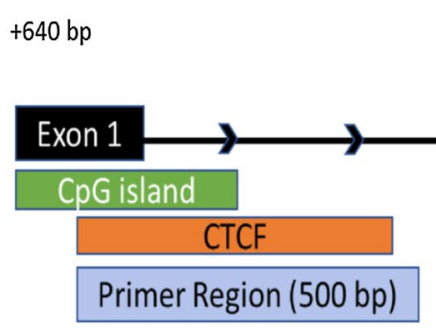

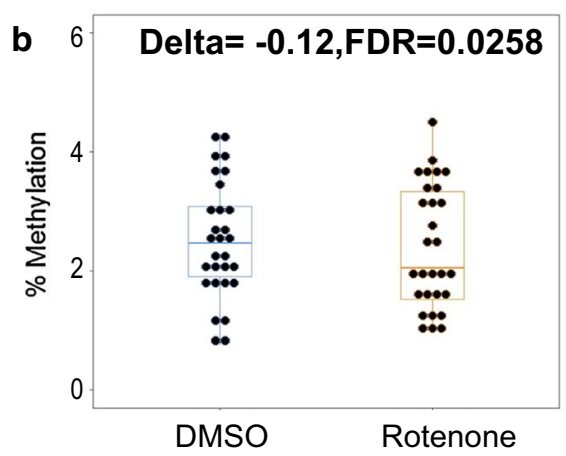

C

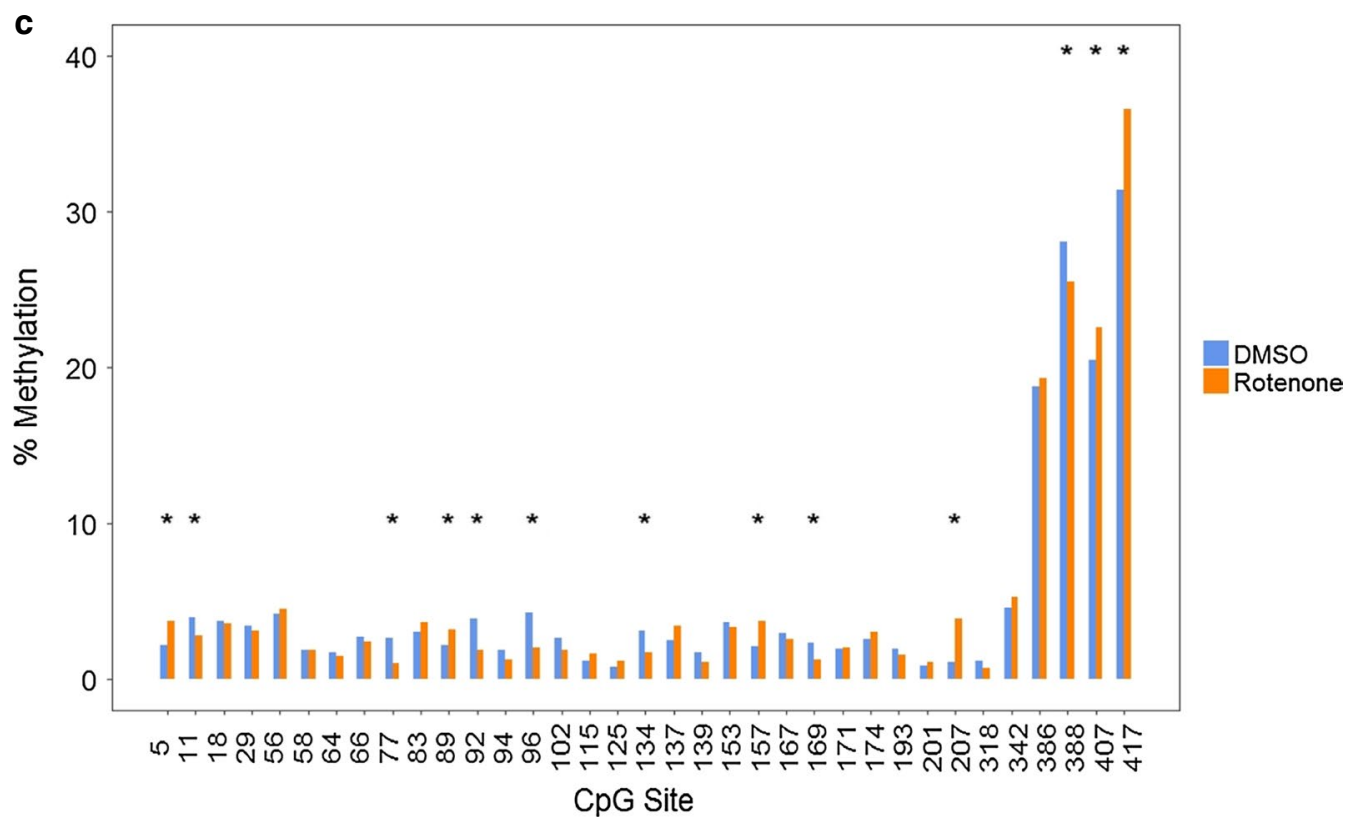

Fig. 6 Altered CpG methylation at NEFM human DNMT1-dependent locus. a Genomic location of the identified NEFM DNMT1-dependent region. The DNA element and distance from the transcription start site are annotated in black. The transcription factor-binding site for CTCF was annotated from ENCODE v2 and ENCODE Uniform TFBS tracks in Genome Browser. The primer region box indicates the amplified region for Bisulfite-sequencing. $\mathbf{b}$ The percent methylation of all CpG sites within the first 200 base-pairs of the amplified region. Delta indicates the change in the mean CpG methylation percentage and the associated false-discovery rate. c The percent methylation of individual $\mathrm{CpG}$ sites within the amplified region. Significant differentially methylated cytosines are indicated by * $(\Delta>1 \%$; $q$ value $<0.01)$

three of these transcription factors were TAF1, TBP, and POL2RA which all have an essential role in initializing transcription. We were interested to see SIN3A and RBBP5 which both interact with histone-modifying enzymes to regulate chromatin accessibility and are critical during neurodevelopment [24]. Furthermore, SIN3A is recruited to the methyl-CpG binding protein $\mathrm{MeCP} 2$ to silence transcription. Mutations in MeCP2 cause an X-linked neurodevelopmental disorder known as Rett Syndrome and similarly impairment of SIN3A expression also causes developmental cognitive deficits [78]. MeCP2 and SIN3A have been linked to the establishment and maintenance of imprinting control regions but their effect on the expression of neighboring imprinted genes remains to be determined [47].

CTCF is another transcription factor of interest with $17 \%$ of the DNMT1-dependent human regions overlapping the CCCTC-binding motif. CTCF is also critical in neurodevelopment and chromatin organization [14, 21]. CTCF mediates the formation of chromatin loops and thus can promote widespread changes in gene expression $[30,58]$. When bound to sequences known as insulator sequences, CTCF represses transcription by blockading promoter-enhancer interactions [7, 27]. CTCF and the stabilizing protein cohesion bind at numerous imprinted control regions $[60,65]$. CTCF has been reported to preferentially bind unmethylated chromatin but binding 
affinity depends not only on the methylation status of the motif itself but the surrounding CpG sites as well [77, 81].

Given the importance of imprinted gene clusters in development, we analyzed functional enrichment of our non-germline ASMs in the human genome. The most significant biological process associated with our gene set was cell to cell adhesion (Fig. 2). We observed a significant group of cadherins at DNMT1-dependent regions on chromosome 5. Cadherin proteins are expressed on the membrane of embryonic stem cells and are critical for their self-renewal by forming tight intracellular niches [59]. The expression of cadherin subtypes on embryonic stem cells is variable and the patterning of cadherin expression also controls their differentiation. Protocadherins are involved in neuronal connectivity and this function extends from neural progenitors during development into postmitotic neurons in the adult brain [66]. Intriguingly, protocadherin is regulated by CTCF and deletion of CTCF in mice caused deficits in hippocampal learning and memory via dysregulation of protocadherin expression [66]. The most significant molecular function was calcium-ion binding and the pre-synaptic axon terminal was one of two most significant cellular components represented. This agreed with our network analyses where multiple genes were involved in cell trafficking and synaptic activity (Fig. 2). We investigated whether developmental genes were specific to an individual tissue or group of tissues. These genes from DNMT1-dependent regions have significant enrichment of genes expressed in the cerebral cortex from two separate databases, EnrichR ArchS4 and Tissue Enrich Human Protein Atlas (Fig. 3). Our data suggest that DNMT1-dependent non-germline ASMs have enhanced expression in the brain, which could be important for neurological development and cellular communication function.

In our previous work, we characterized non-germline ASMs in the mouse genome at two genes, Hcn 2 and Park7, with potential in Parkinson's disease [9, 38, 48]. The proper maintenance of the epigenome throughout aging is believed to have a major impact on the risk of neurodegeneration later in life $[25,40]$. The influence of germline ASMs on neurodegeneration has recently been of interest in the literature given their involvement in neurodevelopment but the effect of non-imprinted ASMs has not been well characterized [25]. To experimentally examine the association of identified ASMs in the human genome with Parkinson's disease, we used human embryonic kidney cells with a neuronal lineage phenotype and treated them with rotenone for $24 \mathrm{~h}$ [69]. We observed several of our candidate genes were affected in response to rotenone treatment and half of these genes have regional expression in Parkinson's disease-associated regions (Fig. 4). Among these genes, HCN2 and NEFM were determined from our tissue enrichment analysis to have a higher expression level in the brain than any other tissue. In addition, experimental analysis of Hcn2 in the mouse genome suggests a random, switchable allelespecific methylation pattern that was independent of the parent-of-origin [48]. We selected these two genes for methylation analysis to determine if conserved non-germline ASMs in the human genome were sensitive to environmental factors associated with Parkinson's disease.

The HCN2 gene encodes an isoform of the hyperpolarization-activated cyclic nucleotide-gated channel located on the membrane of neurons in the central and peripheral nervous system. HCN channels regulate neuronal plasticity and have the advantage of using both voltage dependent mechanisms as well as cAMP intracellular signaling mechanisms [16]. In the midbrain, these channels control the spontaneous activity of dopaminergic neurons and their dysfunction has been linked to the depletion of dopamine in Parkinson's disease [13, 16, 26]. In the human genome (hg19), the conserved DNMT1dependent locus identified was $321 \mathrm{bp}$ at a CpG island on exon 8 of the gene. We observed significant upregulation of mRNA expression levels (1.6-fold change, FDR $<0.01$ ) that correlated with DNA hypomethylation $(-1.8 \%$, FDR $<0.05)$ of a 450 -bp site surrounding the region of interest (Table 2; Fig. 5; Additional file 1: Table S4). Dysregulated $\mathrm{HCN} 2$ expression could affect $\mathrm{HCN} 2$ channel activity leading to disrupted regulation of dopaminergic excitability.

The NEFM gene encodes a subunit of neuron-specific intermediate filaments known as neurofilaments. Neurofilaments are primary components of myelinated axons and are essential for synaptic function [80]. Neurofilament subunit expression is tightly regulated to maintain proper stoichiometry. As such, aberrant expression of NEFM likely disrupts axonal growth and transport. Interestingly, neurofilament subunits including the NEFM protein are considered promising neurodegeneration biomarkers due to their cell specificity and sensitivity to neuronal damage [36]. In Parkinson's disease patients, neurofilament proteins have been detected at higher levels in the cerebral spinal fluid, and more recently, in the blood [1, 63, 64]. In our data, the conserved DNMT1dependent locus covered a 150-bp region in exon 1 as well as a 1650-bp region spanning exon 1 to intron 2 . We observed significant upregulation of mRNA (1.7fold change, FDR $<0.01$ ) and hypomethylation of a 200 bp section of the identified region at exon $1(-0.12 \%$, FDR $<0.05$ ). While the total change in percent methylation was relatively small, the selected region contained a CTCF-binding site. Several CpG sites located within this CTCF-binding motif had higher changes in methylation $(>1 \%$, adjusted $q$ value $<0.01$ ) (Fig. 6; Additional file 1: 
Table S5). As mentioned previously, CTCF is hypersensitive to changes in DNA methylation and approximately $41 \%$ of CTCF-binding variability has been attributed to DNA methylation [77]. The lack of repressive signaling from CTCF could contribute to the observed increases in NEFM reported in Parkinson's disease patients and the observed overexpression of NEFM associated with cytoplasmic inclusions in motor-impaired mice $[45,68,79]$.

We evaluated the recent literature on candidate blood biomarkers in Parkinson's disease patients and observed five differentially methylated genes (COL9A2, SCNN1A, $A M I C A 1, S L C 16 A 3$, and DLK1) in these studies within our conserved human regions $[29,75]$. Of these genes, COL9A2 was differentially expressed in our rotenone treated cells and was determined to have high regional expression in the substantia nigra (Table 2, Fig. 4) [29]. This observation is strengthened with another study that has found that differentially methylated genes in the blood have high concordance with differentially methylated genes in the brain [49]. These data partially support our hypothesis that environmentally induced changes in DNMT1-dependent ASMs in the human brain can alter the risk of neurodegeneration.

\section{Conclusions}

DNMT1 expression in neural stem cells is essential for adult neurogenesis and the survival of adult neurons in the brain [54]. We have shown that non-germline ASMs are dependent on DNMT1 in mice. The goal of this study was to identify conserved DNMT1-dependent regions and putative non-germline ASMs in the human genome and test the vulnerability of these regions to a neurotoxicant associated with Parkinson's disease. Our work identified candidate, non-germline ASMs with DNMT1 dependence as enriched in the human brain. We discovered 14 genes have altered expression ( $>1.5$-fold change) at predicted ASMs in response to rotenone. We quantified methylation of 2 identified regions at adjacent genes (HCN2 and NEFM) known to increase the risk for Parkinson's disease and observed significant hypomethylation. In the future, a larger panel of these identified regions in the human genome will be tested in other cells' lines at varying points in neuronal differentiation to determine the role of non-germline ASMs on neuronal development and its maintenance with age.

\section{Methods}

\section{Identification of conserved DNMT1-dependent regions} in the human genome

Whole Genome Bisulfite Sequencing (WGBS) was used to analyze base resolution methylomes of a series of Dnmt1 (-/-), Dnmt3a (-/-), and Dnmt3b (-/-) murine embryonic stem cell lines (wild-type J1) as described previously [44, 48]. DNMT1-dependent regions termed "NORED" were defined as regions with near complete loss of methylation in Dnmt1 (-/-) compared to wild-type J1 that remained unable to recover methylation after the addition of exogenous Dnmt1 cDNA. To identify the conserved DNMT1-dependent regions in the human genome, we used the UCSC Genome Browser LiftOver software to locate regions in the hg19 assembly from the mouse mm10 assembly. A text file of the chromosome positions (chr: start-end) for each putative ASM was uploaded into LiftOver and converted to the human hg19 assembly with a minimum ratio of 0.1 bases mapping for each region. The genomic location of each conserved region was analyzed in the UCSC Genome Browser window with NCBI RefSeq annotations. Transcription factor binding was observed using the Uniform Transcription Factor Binding data found in the ENCODE Regulation super track. We selected all transcription factor binding sites in H1-human embryonic stem cells (H1-hESCs) detected with CHIP-Seq experiments from the ENCODE consortium from 2007 to 2012 [18]. Imprinted genes from mouse and human genome were identified from the Jirtle Laboratory GeneImprint database (http://www.genei mprint.org/).

\section{Functional enrichment for candidate DNMT1-dependent genes in the human genome}

We restricted functional enrichment analysis of conserved human regions to those that had $>70 \%$ base pair match for over $90 \%$ of the span of the identified region (Table 1). Pathway enrichment and network interactions for human genes nearest to these regions were calculated using the STRING database [71]. Gene Ontology was used for functional annotations and significance was measured using Fisher's exact test with a false-discovery rate $(\mathrm{FDR})<0.05$. Network interactions were clustered using Cytoscape based on gene functional annotations in the reactome pathways [20].

\section{Tissue enrichment for candidate DNMT1-dependent genes} in the human genome

The human DNMT1-dependent genes (Table 1) were used for tissue enrichment in EnrichR [12] using the ArchS4 database [41]. The top six human tissues were reported with a $p$ value $<0.01$ adjusted using their correction for the Fisher's exact test [12]. Enrichment of tissue-specific genes was performed using the TissueEnrich $R$ package which uses gene expression data from the Human Protein Atlas [31]. Genes with an expression level of at least one transcript per million (TPM) were defined as tissue enriched when expression was at least fivefold higher in a distinct tissue compared to the expression 
of any other individual tissue and tissue enhanced when expression was at least fivefold higher in a distinct tissue compared to the total average expression of all other tissues. Group-enriched genes were defined as genes with an expression level of at least one TPM and had at least fivefold higher expression in a group of tissues compared to all tissues. These definitions were taken from TissueEnrich.

\section{Cell culture and treatment of human cell line HEK293}

All media reagents and chemicals in cell culture were purchased from Sigma (St. Louis, MO). Human cell line HEK293 was grown in Dulbecco's Modified Eagle Medium with high glucose, L-glutamine, and sodium pyruvate. Media were supplemented with $10 \%(\mathrm{v} / \mathrm{v})$ heat inactivated fetal bovine serum and 1\% (v/v) PenicillinStreptomycin. HEK293 cells were confirmed by ATCC. Cells were treated at approximately $70 \%$ confluency with $200 \mathrm{nM}$ rotenone or DMSO vehicle control $(<0.001 \%)$ for $24 \mathrm{~h}$. Cell viability was measured with trypan blue $(0.4 \%)$ staining. Viable and dead cells were counted manually using a hematocytometer. A minimum cell viability of $85 \%$ was used for all experiments.

\section{RNA extraction and RNA sequencing library construction}

Total RNA was extracted from two replicates of DMSO or rotenone-treated HEK293 using the trizol method (Invitrogen, Carlsbad, CA). A total of $2 \mu \mathrm{g}$ per sample was used for library construction using the TruSeq Sample Preparation kit from Ilumina (San Diego, CA). Poly-A containing mRNA molecules were isolated from total RNA using oligo-dT attached magnetic beads. Isolated mRNA was then fragmented and synthesized into double-stranded cDNA according to the kit instructions. Ligation of unique Ilumina adapter indices was completed for each sample before bead purification. Libraries were loaded onto a $2 \%$ agarose gel and library products between 200 and $800 \mathrm{bp}$ were purified using the miniElute gel extraction kit from Qiagen (Hilden, Germany). Approximately $150 \mathrm{ng}$ was sent for sequencing on a HiSeq2000 platform with 100 bp paired-end reads.

\section{RNA sequencing data analysis}

Adapter sequences were removed from the raw sequencing data and individual libraries were converted to the fastq format. Sequencing reads were aligned to the human genome (hg19) with TopHat2 (v2.0.9) [37]. For mRNA analyses, the RefSeq database (Build 37.3) was chosen as the annotation references. Read counts of annotated genes were obtained by the Python software HTSeq-count [4]. The read counts of each transcript were normalized to the length of the individual transcript and to the total mapped fragment counts in each sample and expressed as fragments per kilobase of exon per million fragments mapped (FPKM) of mRNAs in each sample. Differentially expressed genes were defined as those with a 1.5-fold change in expression using a false-discovery rate (FDR) $p$ value adjustment $<0.05$ from the edge $R$ package [62]. We examined differentially expressed genes in RNA-seq in common with the genes nearest to the conserved DNMT1-dependent regions in the human genome with $>70 \%$ base-pair match for over $90 \%$ of the span of the identified region in the mouse genome. Overlapping genes were entered into the Genotype-Tissue Expression (GTEx) database using the multi-gene query tool [46]. Parkinson's disease brain regions associated with motor function including the cerebellum, cortex, frontal cortex, spinal cord, substantia nigra, and basal ganglia were selected for further expression analysis.

\section{RNA sequencing validation with quantitative reverse-transcription PCR}

Total RNA was extracted from an additional replicate of HEK293 treated with DMSO or rotenone using the same procedure as stated above. A total of 500 ng RNA was converted to CDNA with the PrimeScript RT reagent kit with gDNA eraser from Takara (Kusatsu, Japan). We selected 7 out of 14 overlapping genes for quantitative PCR (qRT-PCR) analysis using primers listed in Additional file 1: Table S1. All qRT-PCRs were performed on a 7500 Real-Time PCR system from Applied Biosystems (Foster City, CA) using the iTaq Universal SYBR Green Supermix from Bio-Rad (Hercules, CA). The change in expression was normalized to the GAPDH housekeeping gene and expressed as fold change (2- $\left.{ }^{\Delta \Delta \mathrm{CT}}\right)$.

\section{Bisulfite-DNA conversion and Bisulfite-amplicon sequencing library construction}

Genomic DNA was extracted from two replicates of DMSO or rotenone-treated HEK293 using Phenol:Chloroform:Isoamyl alcohol (Sigma, St. Louis, MO). A total of $200 \mathrm{ng}$ DNA was Bisulfite-converted using the Sigma DNA Imprint Modification kit twostep protocol. Bisulfite-converted DNA (BS-DNA) was amplified with primers for selected DNMT1-dependent regions designed with MethPrimer [42] (Additional file 1: Table S2). Amplified BS-DNA products were run on a $2 \% \mathrm{EtBr}$ agarose gel and purified using the mini-Elute gel extraction kit from Qiagen (Hilden, Germany). Purified products for each sample were pooled together and $1 \mathrm{ng}$ was used for library preparation using the Ilumina Nextera DNA Library Preparation kit. Each sample was tagged with a unique Nextera XT adapter (San Diego, CA). Sequencing libraries were quality checked via Bioanalyzer and run on an Ilumina MiSeq platform to generate $150 \mathrm{bp}$ paired end reads. 


\section{Bisulfite-amplicon sequencing analysis}

The raw fastq files were imported into the Galaxy web platform [2]. Reads with quality score $>30$ were trimmed with Trim Galore [39]. Reads were mapped to amplified sequences in the human genome (hg19) using bwa-meth [57]. MethylDackel was used for methylation calling and per-cytosine contexts were merged into per-CPG metrics (https://github.com/dpryan79/MethylDackel). Duplicates and singletons identified in alignment were ignored from the methylation call. Minimum and maximum perbase depths were $1000 \times$ and $100,000 \times$, respectively. The output was selected for methylKit format. Coverage statistics and differentially methylated regions were calculated for CpG sites with methylKit installed in R (v3.5) [3]. Differentially methylated cytosines were defined as being present in both biological replicates, having a minimum absolute difference of $1 \%$ using the coverage weighted mean, and having an SLIM adjusted qvalue $<0.01$ using the methylKit logistic regression model [53]. The change in mean percent methylation $(\Delta \mathrm{me})$ for all $\mathrm{CpG}$ sites within a defined region was calculated by taking the mean number of methylated versus non-methylated $\mathrm{CpG}$ sites from the pooled control and treated samples and using Fisher's exact test FDR $<0.05$.

\section{Supplementary information}

Supplementary information accompanies this paper at https://doi. org/10.1186/s13072-020-00338-8.

Additional file 1: Table S1. qRT-PCR primers for RNAseq validation in human genome. Table S2. BS-PCR primers for human genome. Table S3. BS-PCR primers for human genome. Figure S1. RNA sequencing validation with qRT-PCR. Figure S2. Pearson's correlation coefficient for bisulfite sequencing replicates of HEK293. Figure S3. Bisulfite sequencing coverage of CpG sites within amplified DNMT1-dependent regions at HCN2 and NEFM. Figure S4. Percentage of hyper and hypo differentially methylated CpGs within DNMT1-dependent loci. Table S4. CPG Methylation HCN2. Table S5. CPG Methylation NEFM.

\section{Abbreviations}

PD: Parkinson's disease; DNMT1: DNA methyltransferase l; ASM: Allele-specific methylation; HCN2 (gene): Hyperpolarization Activated Cyclic Nucleotide Gated Potassium And Sodium Channel 2 (protein); NEFM (gene): Neurofilament medium polypeptide (protein); FDR: False-discovery rate; LFC: Log2 fold change; TPM: Transcripts per million.

\section{Acknowledgements}

We also acknowledge JHU Center for Alternatives to Animal Testing for their expertise in cell culture techniques and treatments.

\footnotetext{
Authors' contributions

Conceived concepts for experiments and identified murine ASMs: SNM and ZW. Designed and performed experiments: DMF and DL. Analyzed nextgeneration sequencing data: DMF and YL. Analyzed and interpreted data: DMF and ZW. All authors edited the manuscript. All authors read and approved the final manuscript.

\section{Funding}

This project was made possible with support from the JHU Catalyst Award (ZW) and the student Frederik Bang Award (to DMF). We also acknowledge
}

the National Institutes of Health T32 Pre-doctoral grant (5T32ES7141) for training support (to DMF). Experimental disposals and salaries were supported by the U.S. National Institutes of Health (R01ES25761, U01ES026721 Opportunity Fund, and R21ES028351) to ZW.

\section{Data availability}

The data used to generate this report are available upon request from the corresponding author Dr. Zhibin Wang (zwang47@jhu.edu).

\section{Ethics approval and consent to participate}

Not applicable.

\section{Consent for publication}

Not applicable.

\section{Competing interests}

The authors declare that they have no competing interests.

\section{Author details}

${ }^{1}$ Laboratory of Environmental Epigenomes, Department of Environmental Health \& Engineering, Bloomberg School of Public Health, Johns Hopkins University, Baltimore, MD, USA. ${ }^{2}$ The State Key Laboratory of Biocatalysis and Enzyme Engineering, School of Life Sciences, Hubei University, Wuhan 430062, Hubei, China. ${ }^{3}$ Department of Oncology, School of Medicine, Johns Hopkins University, Baltimore, MD, USA.

Received: 8 December 2019 Accepted: 6 March 2020

Published online: 16 March 2020

\section{References}

1. Abdo WF, Bloem BR, Van Geel WJ, Esselink RA, Verbeek MM. CSF neurofilament light chain and tau differentiate multiple system atrophy from Parkinson's disease. Neurobiol Aging. 2007;28(5):742-7.

2. Afgan E, Baker D, Van den Beek M, Blankenberg D, Bouvier D, Čech $M$, et al. The galaxy platform for accessible, reproducible and collaborative biomedical analyses: 2016 update. Nucleic Acids Res. 2016:44(W1):W3-10.

3. Akalin A, Kormaksson M, Li S, Garrett-Bakelman FE, Figueroa ME, Melnick A, et al. methylKit: a comprehensive $\mathrm{R}$ package for the analysis of genome-wide DNA methylation profiles. Genome Biol. 2012;13(10):R87.

4. Anders S, Pyl PT, Huber W. HTSeq-a python framework to work with high-throughput sequencing data. Bioinformatics. 2015;31(2):166-9.

5. Barlow DP, Bartolomei MS. Genomic imprinting in mammals. Cold Spring Harb Perspect Biol. 2014. https://doi.org/10.1101/cshperspect.a018382.

6. Bartolomei MS, Tilghman SM. Genomic imprinting in mammals. Annu Rev Genet. 1997;31(1):493-525.

7. Bell AC, Felsenfeld G. Methylation of a CTCF-dependent boundary controls imprinted expression of the lgf2 gene. Nature. 2000;405(6785):482.

8. Benton MC, Lea RA, Macartney-Coxson D, Sutherland HG, White N, Kennedy D, Mengerson K, Haupt LM, Griffiths LR. Genome-wide allelespecific methylation is enriched at gene regulatory regions in a multigeneration pedigree from the Norfolk Island isolate. Epigenet Chromatin. 2019. https://doi.org/10.1186/s13072-019-0304-7.

9. Bonifati V, Rizzu P, van Baren MJ, Schaap O, Breedveld GJ, Krieger E, et al. Mutations in the DJ-1 gene associated with autosomal recessive early-onset parkinsonism. Science. 2003;299(5604):256-9. https://doi. org/10.1126/science.1077209.

10. Butler MG. Genomic imprinting disorders in humans: a mini-review. J Assist Reprod Genet. 2009;26(9-10):477-86.

11. Chen T, Hevi S, Gay F, Tsujimoto N, He T, Zhang B, Ueda Y, Li E. Complete inactivation of DNMT1 leads to mitotic catastrophe in human cancer cells. Nat Genet. 2007;39:391-6.

12. Chen EY, Tan CM, Kou Y, Duan Q, Wang Z, Meirelles GV, et al. Enrichr: interactive and collaborative HTML5 gene list enrichment analysis tool. BMC Bioinform. 2013;14(1):128.

13. Chen L, Xu R, Sun F, Xue Y, Hao X, Liu H, et al. Hyperpolarization-activated cyclic nucleotide-gated ( $\mathrm{HCN}$ ) channels regulate firing of globus pallidus neurons in vivo. Mol Cell Neurosci. 2015;68:46-55. 
14. Davis L, Onn I, Elliott E. The emerging roles for the chromatin structure regulators CTCF and cohesin in neurodevelopment and behavior. Cell Mol Life Sci. 2018;75(7):1205-14.

15. Deng Q, Ramskold D, Reinius B, Sandberg R. Single-cell RNA-seq reveals dynamic, random monoallelic gene expression in mammalian cells. Science. 2014;343(6167):193-6. https://doi.org/10.1126/science.1245316.

16. DiFrancesco D. Dual allosteric modulation of pacemaker (f) channels by CAMP and voltage in rabbit SA node. J Physiol (Lond). 1999;515(2):367-76.

17. Dolinoy DC, Weidman JR, Waterland RA, Jirtle RL. Maternal genistein alters coat color and protects avy mouse offspring from obesity by modifying the fetal epigenome. Environ Health Perspect. 2006;114(4):567-72. https ://doi.org/10.1289/ehp.8700.

18. ENCODE Project Consortium. An integrated encyclopedia of DNA elements in the human genome. Nature. 2012;489(7414):57.

19. Esumi S, Kakazu N, Taguchi Y, Hirayama T, Sasaki A, Hirabayashi T, et al. Monoallelic yet combinatorial expression of variable exons of the protocadherin-a gene cluster in single neurons. Nat Genet. 2005;37(2):171-6.

20. Fabregat A, Korninger F, Viteri G, Sidiropoulos K, Marin-Garcia P, Ping P, et al. Reactome graph database: efficient access to complex pathway data. PLoS Comput Biol. 2018;14(1):e1005968.

21. Franco MM, Prickett AR, Oakey RJ. The role of CCCTC-binding factor (CTCF) in genomic imprinting, development, and reproduction. Biol Reprod. 2014; 91(5):125, 1-9.

22. Freeman DM, Wang Z. Towards the molecular mechanisms of transgenerational epigenetic inheritance: insights from transgenic mice. Transgenerational epigenetics. New York: Academic Press; 2019. p. 137-56.

23. Freeman DM, Lou D, Li Y, Martos SN, Wang Z. The conserved DNMT1 dependent methylation regions in human cells are vulnerable to environmental rotenone. bioRxiv; 2019. 798587.

24. Gabriele M, Tobon AL, D'Agostino G, Testa G. The chromatin basis of neurodevelopmental disorders: rethinking dysfunction along the molecular and temporal axes. Prog Neuro-Psychopharmacol Biol Psychiatry. 2018;84:306-27

25. Gapp K, Woldemichael BT, Bohacek J, Mansuy IM. Epigenetic regulation in neurodevelopment and neurodegenerative diseases. Neuroscience. 2014;264:99-111.

26. Good CH, Hoffman AF, Hoffer BJ, Chefer VI, Shippenberg TS, Bäckman $\mathrm{CM}$, et al. Impaired nigrostriatal function precedes behavioral deficits in a genetic mitochondrial model of parkinson's disease. FASEB J. 2011;25(4):1333-44.

27. Hark AT, Schoenherr CJ, Katz DJ, Ingram RS, Levorse JM, Tilghman SM CTCF mediates methylation-sensitive enhancer-blocking activity at the H19/lgf2 locus. Nature. 2000;405(6785):486

28. Harris G, Eschment M, Orozco SP, McCaffery JM, Maclennan R, Severin $D$, et al. Toxicity, recovery, and resilience in a 3D dopaminergic neuronal in vitro model exposed to rotenone. Arch Toxicol. 2018:92(8):2587-606.

29. Henderson-Smith A, Fisch KM, Hua J, Liu G, Ricciardelli E, Jepsen K, et al. DNA methylation changes associated with Parkinson's disease progression: outcomes from the first longitudinal genome-wide methylation analysis in blood. Epigenetics. 2019;14(4):365-82.

30. Hou C, Zhao H, Tanimoto K, Dean A. CTCF-dependent enhancer-blocking by alternative chromatin loop formation. Proc Natl Acad Sci USA. 2008;105(51):20398-403. https://doi.org/10.1073/pnas.0808506106.

31. Jain A, Tuteja G. TissueEnrich: tissue-specific gene enrichment analysis. Bioinformatics. 2018;35(11):1966-7.

32. Jirtle RL, Skinner MK. Environmental epigenomics and disease susceptibility. Nat Rev Genet. 2007;8(4):253.

33. Kacem $S$, Feil R. Chromatin mechanisms in genomic imprinting. Mamm Genome. 2009;20(9-10):544-56.

34. Kappil M, Lambertini L, Chen J. Environmental influences on genomic imprinting. Curr Environ Health Rep. 2015;2(2):155-62.

35. Kerkel K, Spadola A, Yuan E, Kosek J, Jiang L, Hod E, et al. Genomic surveys by methylation-sensitive SNP analysis identify sequence-dependent allele-specific DNA methylation. Nat Genet. 2008;40(7):904.

36. Khalil M, Teunissen CE, Otto M, Piehl F, Sormani MP, Gattringer T, et al. Neurofilaments as biomarkers in neurological disorders. Nat Rev Neurol. 2018;14(10):577-89.

37. Kim D, Pertea G, Trapnell C, Pimentel H, Kelley R, Salzberg SL. TopHat2: accurate alignment of transcriptomes in the presence of insertions, deletions and gene fusions. Genome Biol. 2013;14(4):R36.
38. Kim RH, Smith PD, Aleyasin H, Hayley S, Mount MP, Pownall S, et al. Hypersensitivity of DJ-1-deficient mice to 1-methyl-4-phenyl-1, 2, 3, 6-tetrahydropyrindine (MPTP) and oxidative stress. Proc Natl Acad Sci. 2005;102(14):5215-20.

39. Krueger F. Trim galore. A wrapper tool around Cutadapt and FastQC to consistently apply quality and adapter trimming to FastQ files. 2015. http://www.bioinformatics.babraham.ac.uk/projects/trim_galore/

40. Labbé C, Lorenzo-Betancor O, Ross OA. Epigenetic regulation in Parkinson's disease. Acta Neuropathol. 2016;132(4):515-30.

41. Lachmann A, Torre D, Keenan AB, Jagodnik KM, Lee HJ, Wang L, et al. Massive mining of publicly available RNA-seq data from human and mouse. Nat Commun. 2018;9(1):1366.

42. Li L, Dahiya R. MethPrimer: designing primers for methylation PCRs. Bioinformatics. 2002;18(11):1427-31.

43. Li W, Qin W, Liu H, Fan L, Wang J, Jiang T, et al. Subregions of the human superior frontal gyrus and their connections. Neuroimage. 2013;78:46-58.

44. Li Z, Dai H, Martos SN, Xu B, Gao Y, Li T, et al. Distinct roles of DNMT1dependent and DNMT1-independent methylation patterns in the genome of mouse embryonic stem cells. Genome Biol. 2015;16(1):115.

45. Liu Q, Xie F, Alvarado-Diaz A, Smith MA, Moreira PI, Zhu X, et al. Neurofilamentopathy in neurodegenerative diseases. Open Neurol J. 2011;5:58-62. https://doi.org/10.2174/1874205x01105010058.

46. Lonsdale J, Thomas J, Salvatore M, Phillips R, Lo E, Shad S, et al. The genotype-tissue expression (GTEx) project. Nat Genet. 2013;45(6):580

47. Ma P, de Waal E, Weaver JR, Bartolomei MS, Schultz RM. A DNMT3A2HDAC2 complex is essential for genomic imprinting and genome integrity in mouse oocytes. Cell Rep. 2015;13(8):1552-60.

48. Martos SN, Li T, Ramos RB, Lou D, Dai H, Xu J, et al. Two approaches reveal a new paradigm of 'switchable or genetics-influenced allele-specific DNA methylation with potential in human disease. Cell Discov. 2017;3:17038.

49. Masliah E, Dumaop W, Galasko D, Desplats P. Distinctive patterns of DNA methylation associated with Parkinson disease: identification of concordant epigenetic changes in brain and peripheral blood leukocytes. Epigenetics. 2013;8(10):1030-8.

50. Monteagudo-Sánchez A, Sánchez-Delgado M, Guara Ciurana S, Medrano J, Poo-Llanillo ME, Ishida M et al. Epigenetic asymmetry of DLGAP2: Preimplantation maternal methylation switches to a random monoallelic profile in somatic tissues. OBM genetics. 2018. https://doi.org/10.21926/ obm.genet.1803026

51. Murphy SK, Hoyo C. Sculpting our future: environmental nudging of the imprintome. Environmental epigenomics in health and disease. Berlin: Springer; 2013. p. 51-73.

52. Navarro-Sánchez L, Águeda-Gómez B, Aparicio S, Pérez-Tur J. Epigenetic study in Parkinson's disease: a pilot analysis of DNA methylation in candidate genes in brain. Cells. 2018;7(10):150.

53. Ning Y, Yang J, Ma G, Chen P. Modelling rock blasting considering explosion gas penetration using discontinuous deformation analysis. Rock Mech Rock Eng. 2011;44(4):483-90.

54. Noguchi H, Kimura A, Murao N, Matsuda T, Namihira M, Nakashima K. Expression of DNMT1 in neural stem/precursor cells is critical for survival of newly generated neurons in the adult hippocampus. Neurosci Res. 2015;95:1-11.

55. Onuchic V, Lurie E, Carrero I, Pawliczek P, Patel RY, Rozowsky J, et al. Allele-specific epigenome maps reveal sequence-dependent stochastic switching at regulatory loci. Science. 2018. https://doi.org/10.1126/scien ce.aar3146 (Epub 2018 Aug 23).

56. Orth M, Tabrizi S, Schapira A, Cooper J. a-Synuclein expression in HEK293 cells enhances the mitochondrial sensitivity to rotenone. Neurosci Lett. 2003;351(1):29-32.

57. Pedersen BS, Eyring K, De S, Yang IV, Schwartz DA. 2014. Fast and accurate alignment of long bisulfite-seq reads. arXiv preprint arXiv:1401.1129.

58. Phillips JE, Corces VG. CTCF: master weaver of the genome. Cell. 2009;137(7):1194-211.

59. Pieters T, van Roy F. Role of cell-cell adhesion complexes in embryonic stem cell biology. J Cell Sci. 2014;127(Pt 12):2603-13. https://doi. org/10.1242/jcs.146720.

60. Prickett AR, Barkas N, McCole RB, Hughes S, Amante SM, Schulz R, et al. Genome-wide and parental allele-specific analysis of CTCF and cohesin DNA binding in mouse brain reveals a tissue-specific binding pattern and an association with imprinted differentially methylated regions. Genome Res. 2013;23(10):1624-35. https://doi.org/10.1101/gr.150136.112. 
61. Rakyan VK, Chong S, Champ ME, Cuthbert PC, Morgan HD, Luu KV, et al. Transgenerational inheritance of epigenetic states at the murine axin(fu) allele occurs after maternal and paternal transmission. Proc Natl Acad Sci USA. 2003;100(5):2538-43. https://doi.org/10.1073/pnas.0436776100.

62. Robinson MD, McCarthy DJ, Smyth GK. edgeR: a bioconductor package for differential expression analysis of digital gene expression data. Bioinformatics. 2010;26(1):139-40.

63. Rojas JC, Karydas A, Bang J, Tsai RM, Blennow K, Liman V, et al. Plasma neurofilament light chain predicts progression in progressive supranuclear palsy. Ann Clin Transl Neurol. 2016;3(3):216-25.

64. Rosengren LE, Karlsson J, Karlsson J, Persson LI, Wikkelsø C. Patients with amyotrophic lateral sclerosis and other neurodegenerative diseases have increased levels of neurofilament protein in CSF. J Neurochem 1996;67(5):2013-8.

65. Rubio ED, Reiss DJ, Welcsh PL, Disteche CM, Filippova GN, Baliga NS, et al. CTCF physically links cohesin to chromatin. Proc Natl Acad Sci USA. 2008;105(24):8309-14. https://doi.org/10.1073/pnas.0801273105.

66. Sams DS, Nardone S, Getselter D, Raz D, Tal M, Rayi PR, et al. Neuronal CTCF is necessary for basal and experience-dependent gene regulation, memory formation, and genomic structure of BDNF and arc. Cell Rep. 2016;17(9):2418-30

67. Sang L, Zhang J, Wang L, Zhang J, Zhang Y, Li P, et al. Alteration of brain functional networks in early-stage Parkinson's disease: a resting-state fMRI study. PLoS ONE. 2015;10(10):e0141815.

68. Sosa MAG, Friedrich VL Jr, DeGasperi R, Kelley K, Wen PH, Senturk E, et al. Human midsized neurofilament subunit induces motor neuron disease in transgenic mice. Exp Neurol. 2003;184(1):408-19.

69. Stepanenko A, Dmitrenko V. HEK293 in cell biology and cancer research: phenotype, karyotype, tumorigenicity, and stress-induced genomephenotype evolution. Gene. 2015;569(2):182-90.

70. Susiarjo M, Sasson I, Mesaros C, Bartolomei MS. Bisphenol a exposure disrupts genomic imprinting in the mouse. PLoS Genet. 2013;9(4):e1003401.

71. Szklarczyk D, Franceschini A, Wyder S, Forslund K, Heller D, Huerta-Cepas J, et al. STRING v10: protein-protein interaction networks, integrated over the tree of life. Nucleic Acids Res. 2014;43(D1):D447-52.

72. Tanner CM, Kamel F, Ross GW, Hoppin JA, Goldman SM, Korell M, et al. Rotenone, paraquat, and Parkinson's disease. Environ Health Perspect. 2011;119(6):866-72. https://doi.org/10.1289/ehp.1002839.

73. Teixeira J, Basit F, Swarts HG, Forkink M, Oliveira PJ, Willems PH, et al. Extracellular acidification induces ROS-and mPTP-mediated death in HEK293 cells. Redox Biol. 2018;15:394-404.

74. Walsh CP, Chaillet JR, Bestor TH. Transcription of IAP endogenous retroviruses is constrained by cytosine methylation. Nat Genet. 1998;20:116-7.
75. Wang C, Chen L, Yang Y, Zhang M, Wong G. Identification of potential blood biomarkers for Parkinson's disease by gene expression and DNA methylation data integration analysis. Clin Epigenet. 2019;11(1):24.

76. Wang H, Lou D, Wang Z. Crosstalk of genetic variants, allele-specific DNA methylation, and environmental factors for complex disease risk. Front Genet. 2018;9:695.

77. Wang H, Maurano MT, Qu H, Varley KE, Gertz J, Pauli F, et al. Widespread plasticity in CTCF occupancy linked to DNA methylation. Genome Res. 2012:22(9):1680-8. https://doi.org/10.1101/gr.136101.111.

78. Witteveen JS, Willemsen MH, Dombroski TC, Van Bakel NH, Nillesen WM, Van Hulten JA, et al. Haploinsufficiency of MeCP2-interacting transcriptional co-repressor SIN3A causes mild intellectual disability by affecting the development of cortical integrity. Nat Genet. 2016;48(8):877.

79. Wong PC, Marszalek J, Crawford TO, Xu Z, Hsieh ST, Griffin JW, et al. Increasing neurofilament subunit NF-M expression reduces axonal NF-H, inhibits radial growth, and results in neurofilamentous accumulation in motor neurons. J Cell Biol. 1995;130(6):1413-22. https://doi.org/10.1083/ jcb.130.6.1413.

80. Yuan A, Rao MV, Veeranna Nixon RA. Neurofilaments and neurofilament proteins in health and disease. Cold Spring Harb Perspect Biol. 2017. https://doi.org/10.1101/cshperspect.a018309.

81. Li Y, Huang W, Niu L, Umbach DM, Covo S, Li L. Characterization of constitutive CTCF/cohesin loci: a possible role in establishing topological domains in mammalian genomes. BMC Genomics. 2013;14(1):553

82. Zhang Y, Rohde C, Reinhardt R, Voelcker-Rehage C, Jeltsch A. Nonimprinted allele-specific DNA methylation on human autosomes. Genome Biol. 2009:10(12):R138.

83. Zhou W, Bouhassira EE, Tsai HM. An IAP retrotransposon in the mouse ADAMTS13 gene creates ADAMTS13 variant proteins that are less effective in cleaving von willebrand factor multimers. Blood. 2007:110(3):886-93.

84. Ziebarth JD, Bhattacharya A, Cui Y. CTCFBSDB 2.0: a database for CTCF-binding sites and genome organization. Nucleic Acids Res. 2012;41(D1):D188-94

85. Zuin J, Dixon JR, van der Reijden MI, Ye Z, Kolovos P, Brouwer RW, et al. Cohesin and CTCF differentially affect chromatin architecture and gene expression in human cells. Proc Natl Acad Sci USA. 2014;111(3):996-1001. https://doi.org/10.1073/pnas.1317788111.

\section{Publisher's Note}

Springer Nature remains neutral with regard to jurisdictional claims in published maps and institutional affiliations.
Ready to submit your research? Choose BMC and benefit from:

- fast, convenient online submission

- thorough peer review by experienced researchers in your field

- rapid publication on acceptance

- support for research data, including large and complex data types

- gold Open Access which fosters wider collaboration and increased citations

- maximum visibility for your research: over $100 \mathrm{M}$ website views per year

At BMC, research is always in progress.

Learn more biomedcentral.com/submissions 